\title{
Real options in strategic investment games between two asymmetric firms
}

\author{
Jean J. KONG and Yue Kuen KWOK*
}

July 24, 2006

\author{
Department of Mathematics \\ Hong Kong University of Science and Technology \\ Clear Water Bay, Hong Kong
}

\begin{abstract}
This paper examines strategic investment games between two firms that compete for optimal entry in a project that generates uncertain revenue flows. Under asymmetry on both the sunk cost of investment and revenue flows of the two competing firms, we investigate the value of real investment options and strategic interaction of investment decisions. Compared to earlier models that only allow for asymmetry on sunk cost, our model demonstrates a richer set of strategic interactions of entry decisions. We provide a complete characterization of pre-emptive, dominant and simultaneous equilibriums by analyzing the relative value of leader's and follower's optimal investment thresholds. In a duopoly market with negative externalities, a firm may reduce loss of real options value by selecting appropriate pre-emptive entry. When one firm has a dominant advantage over its competitor, both the dominant firm and dominated firm enter at their respective leader's and follower's optimal thresholds. When the pre-emptive thresholds of both firms happen to coincide, the two firms enter simultaneously. Under positive externalities, firms do not compete to lead.
\end{abstract}

Keywords: Real options, strategic investment games, pre-emption

JEL classifications: G31, D81, O31

\footnotetext{
*Correspondence author; email: maykwok@ust.hk
} 


\section{Introduction}

The real world of investment is characterized by strategic competition among rival firms, where each firm makes assessment on its own strategic competitiveness among competing parties. Market conditions such as current product value and volatility of revenue flows, and firm specific factors such as sunk cost and market share in monopoly and duopoly states directly affect the outcomes of strategic competition. Also, externalities can induce quite different entry decisions between two competing firms. Positive externalities mean returns in duopoly state exceed that in monopoly state, and vice versa for negative externalities. A possible cause of positive externalities could be mutual learning gain, which leads to product improvement and consumption growth. Under positive externalities, firms do not compete to lead. Negative externalities are more common in practice, as firms always undercut one another with new entry. Negative externalities combined with asymmetric firms allow for a richer set of strategic interaction of entry decisions. The pre-emptive decision made by any competing firms should be in consistence with game equilibrium. In any strategic investment models, individual firm's optimal entry thresholds are determined with reference to strategic competitiveness of the firm. In this paper, we attempt to quantify strategic competitiveness and explore the nature of various types of competitive equilibriums.

Traditionally, investment decisions are analyzed based on the net present value (NPV) model. The NPV of an investment is calculated from its discounted cash flow of future earnings, which are taken to be known at the current moment. The deterministic nature of NPV fails to accommodate market uncertainty, irreversibility of investment and ability to delay entry. All of the above factors constitute a value of waiting. The real options approach incorporates this value of waiting when determining an optimal entry point. Specifically, the real option analysis exploits the analogy between a firm's investment opportunity and a financial call option (Dixit and Pindyck, 1994).

The analysis of strategic investment decisions under the joint framework of real options and game theoretic setting has been the subject of intense research interests. A recent survey of real options and strategic competition is presented by Boyer et al. (2004). Fudenberg and Tirole (1985) presents the first strategic investment model that analyzes the effects of pre-emption in games of timing in the adoption of a new technology by two competing firms. 
Though their model assumes zero market uncertainty, their technique of relating the threat of pre-emption to rent equalization forms the cornerstone of analysis of strategic investment model. Rent equalization is a state equilibrium at which the benefit of being the leader equals that of the follower. In a subsequent work, Grenadier (1996) seeks to explain the property market's boom and bust cycle using competition between symmetric firms. He uses an equilibrium framework for strategic option exercise games to model the timing of real estate development. His model predicts that markets with a longer time-to-build will display a greater propensity towards building booms in the face of declining demand and property values. Empirical evidence on overbuilding at the end of a property boom underlines the risk of pre-emption. The fear of being pre-empted forces firms to invest non-optimally and subsequently depresses their combined returns. Grenadier's paper also points out the dual characteristics of volatility in real options. On the one hand, increasing volatility tends to postpone investment. And on the other hand, rising volatility may make it likely that substantial increase in demand is achieved sooner.

Mason and Weeds (2005) show that when two identical firms compete non-cooperatively, a firm must pre-empt at lower than optimal entry point to guarantee leadership. They also find that pre-emptive threshold may drop in the face of increasing uncertainty (volatility). In a duopoly market involving symmetric firms, a firm's entry point is influenced by two opposite forces: the desire to wait for optimal entry and the urge to pre-empt. Their paper examines how investment decisions are dictated by uncertain returns, pre-emption threat and a range of positive and negative externalities. They show that pre-emption threat can totally erase the option value of waiting imparted by irreversibility and uncertainty. In addition, they discuss the various investment inefficiencies caused by externalities.

A firm's competitive advantage is modeled in Joaquin and Butler (1999) through a deterministic cost-revenue asymmetry. When the state variable, which embodies market uncertainty, is initialized at a sub-optimal level, the low-cost firm always leads due to its dominant competitive advantage. Their paper also considers the scenario when the initial market conditions are above the entry thresholds. In this case, immediate entry for both firms is justified. There can be three possible entry equilibriums: low-cost firm leads, high-cost firm leads and simultaneous entry of both firms.

In an attempt to explain the undeveloped market boom marked by simultaneous entry, Huisman et al. (2004) and Pawlina and Kort (2006) examine 
the effect of first mover advantage in combination with cost asymmetry. They find that when asymmetry is relatively small and first-mover advantage is not significant, simultaneous entry may be favored. Their results demonstrate how cost asymmetry may induce keen competition. When the firms do not face adverse negative externalities (that is, subsequent entry of the second mover does not cause great reduction in the revenue flow of the first entrant), introducing a small amount of cost asymmetry may induce simultaneous entry. When the first mover premium is large, like that in the technology sector, their model shows that simultaneous entry will not commence.

Huisman (2001) presents detailed studies of a dynamic duopoly market where two asymmetric firms compete in the adoption of new technology. Three types of equilibriums can be identified. The first type is sequential equilibrium, which occurs when cost asymmetry is high so that the low-cost firm has dominant competitive advantage. In this case, the leader acts like a monopolist and will enter at its optimal leader threshold. The rival firm is restrained from pre-emption due to its high cost to entry. The second type is pre-emptive equilibrium, which occurs when cost asymmetry is not substantial. In this case, both firms have the incentive to pre-empt. In order to secure the first mover advantage, the first entrant must enter at a nonoptimal point. The last type is simultaneous investment equilibrium, where both parties rush to enter at the same threshold level. Our analysis presented in this paper shows that the simultaneous investment equilibrium can never occur under cost asymmetry alone. In other words, simultaneous entry may occur only when the two firms have asymmetry both in cost and revenue.

Pawlina (2003) compares social welfare across different outcomes of strategic competition and examines how to design suitable policies so as to maximize social welfare. In his paper, social welfare is gauged by weighing social surplus against loss in firm value due to early investment. If overall cost of investment to both firms is small, then the loss of firm value is viewed as a fair sacrifice for higher social surplus since this will bring better quality product to consumer earlier at a small cost to the firms. In this way, any policy that favors pre-emptive or sequential entry equilibriums will benefit consumers. If overall cost of investment to both firms is high, the gain in social surplus will never fully compensate for the cost of an early investment. Therefore, narrowing of cost asymmetry to induce simultaneous entry is preferred. In other words, competitive advantage of one firm over its rival should be minimized in a high investment cost environment.

In this paper, we analyze the entry decisions of competing firms in a 
duopoly real option game on an irreversible investment project that generates uncertain revenue returns. We extend the earlier duopoly models by allowing asymmetry in both sunk cost of investment and revenue flows. With asymmetry on sunk cost alone, the lower-cost firm will always lead. Given that both firms are receiving the same revenue flow, it is not surprising that the low-cost firm always enjoys competitive advantage over its high-cost rival. Simultaneous entry is ruled out completely if the initial value of the state variable renders both leader value functions below the corresponding follower value functions. With asymmetry on both cost and revenue, simultaneous entry or pre-emption by the high-cost firm is now possible, assuming the same initial conditions above. In particular, simultaneous entry occurs when the pre-emptive thresholds of the two firms coincide. Firms do pursue such a lose-lose strategy, and this phenomenon is coined "non-zero probability of mistake" in real options literature. The phenomenon is attributed to the non-zero intensity of entry at the pre-emptive threshold under asymmetry, unlike similar occurrence of simultaneous entry in the case of symmetric firms discussed by Fudenberg and Tirole (1985). Furthermore, our analysis indicates that the combination of firm's competitiveness relative to the states (duopoly or monoploy) and to its rival is crucial to determining the strategic equilibriums in a lose-lose competition.

Our model resembles the new market model in Huisman's paper (2001). In addition, we introduce revenue asymmetry to allow for variation in relative competitiveness. Under cost asymmetry alone, the low-cost firm enjoys competitive advantage over its rival throughout. However, this would not be the case when there is also asymmetry in revenue. In this paper, we extend earlier strategic investment game models by providing a complete analysis of strategic equilibriums under asymmetry in both sunk cost and revenue. The structure of this paper is organized as follows. In the next section, we present the formulation of the two-firm strategic investment model under sunk cost and revenue asymmetry. The important parameters in the model are the firm costs normalized by the revenue flows in the respective monopoly and duopoly states. All optimal investment threshold values in our real game option model are seen to be multiple of these parameter values. In Section 3 , we derive the value functions and entry thresholds under three scenarios, where the firm may serve as the follower, pre-emptive leader or dominant leader. In Section 4, we establish analytic results for the existence of preemptive thresholds and the relative magnitude of the pre-emptive thresholds. We show that under positive enternalities, pre-emptive threshold never exists 
while pre-emptive incentive of at least one firms exist under negative externalities. When the pre-emptive thresholds of both firms exist, we perform theoretical analysis on the characterization of the relative magnitude of the two thresholds. In Section 5, we consider strategic equilibriums under both negative and positive externalities and the strategic investment decisions of the two firms under various forms of strategic equilibriums, which include the sequential entry equilibrium, pre-emptive entry equilibrium and simultaneous entry equilibrium. Summary and conclusive remarks are presented in the last section.

\section{Two-firm strategic investment model}

We consider two firms that compete in an investment project to gain entry into a product market. Both firms have the option to wait for their optimal entry into the market. Further, we make the assumption of perpetuality such that the investment opportunity lasts forever, that is, the investors can choose to wait for optimal entry indefinitely. The investment decision is irreversible and the sunk cost of investment are asymmetric between the two firms. There are no future variable costs of production after investment. The two firms are assumed to be value maximizing and each firm can choose to lead, follow or enter simultaneously in timing its investment entry. The market is said to be in monopoly state or duopoly state, according to whether one firm or two firms are operating, respectively. The revenue flows after entry depend on the number of operating firms in the market (monopoly or duopoly) and the identity of the firm. The stochastic nature of the revenue flows are modeled by assuming that they take the form of a constant multiple of the Geometric Brownian process $\theta_{t}, t \geq 0$, where

$$
d \theta_{t}=\mu \theta_{t} d t+\sigma \theta_{t} d Z_{t} .
$$

Here, the state variable $\theta_{t}$ may be considered as the underlying stochastic aggregate economic factors affecting the revenue and $Z_{t}$ is the standard Brownian process. The volatility $\sigma$ and drift rate $\mu$ are assumed to be constant. To ensure finite valuation of perpetual stream of revenue flows, it is necessary to impose the condition: $\mu<r$, where $r$ is the riskless interest rate. Let $m$ and $d$ denote the monopoly and duopoly state, respectively. The instantaneous revenue flow for firm $i$ at state $j$ is given by

$$
\pi_{t}^{i j}=D_{i j} \theta_{t}
$$


where $D_{i j}$ is a constant multiplier, $i \in\{1,2\}$ and $j \in\{m, d\}$. The firms are said to face negative externalities when

$$
0<D_{i d}<D_{i m}, \quad i=1,2 .
$$

or positive externalities when

$$
0<D_{i m}<D_{i d}, \quad i=1,2 .
$$

Negative (positive) externalities mean lower (higher) revenue flows when both firms are operating in the same product market. It will be shown later that the case of negative externalities may induce keen competition between the two firms. In particular, when there is no clear comparative advantage of one firm over the other as the leader, the two firms would be both worse off by rushing to pre-empt in the so-called lose-lose competition. In our analysis, we assume the current value of the stochastic state variable $\theta_{0}$ to be sufficiently low ( $\theta_{0}$ is below all optimal investment thresholds for both firms) so that both firms have to wait before making their investment entries. Our twofirm model assumes asymmetry both in sunk cost of investment and revenue flows. We let $K_{i}$ denote the sunk cost of Firm $i, i \in\{1,2\}$, and define the revenue adjusted cost $\widetilde{K}_{i j}$ by

$$
\widetilde{K}_{i j}=K_{i} / D_{i j}, \quad i \in\{1,2\}, j \in\{m, d\} .
$$

It will be shown later that the optimal investment threshold values in our real options model and the Marshallian threshold values under the "net present value" rule are constant multiples of $\widetilde{K}_{i j}$. Compared to earlier models [Huisman (2001), Kijima and Shibata (2002), Pawlina (2003), Mason and Weeds (2005)], our model has a higher level of generality under the assumption of asymmetry in both cost and revenue, thus generates a richer set of strategic equilibriums.

Taking Firm 1 as an example, there are three possible choices of investment timing. It may serve as the leader which invests before its competitor (Firm 2) or as the follower, or it enters into the product market simultaneously with Firm 2. In the solution procedure, which is typical of dynamic games, the problem is solved backward. We first solve for the follower value function and the corresponding investment threshold, then followed by solution of the value function and investment threshold of the leader. When both firms invest simultaneously, their value functions are simply given by the respective follower functions. 


\section{Value functions and investment thresholds}

In this section, we employ the standard Bellman's optimality argument to derive the value functions and investment thresholds of Firm $i, i=1,2$. We denote the rival firm as Firm $i^{\prime}$ so that $i^{\prime}=2$ when $i=1$ and vice versa. We consider the value functions under three scenarios, namely, the follower value function, the pre-emptive leader's value function and the leader value function under non-competitive status. We also derive the optimal investment threshold at which the firm may follow, pre-empt or dominate. These threshold values play the key role in determining whether the firms should take pre-emptive action, dominate or simultaneously enter into the product market. The value functions have different functional representations over different ranges of $\theta$, depending on the investment status of the firm itself and its competitor.

Under the uncertain revenue processes, the optimal time of investment is random. Let $(\Omega, \mathcal{F}, P)$ denote the relevant probability space of our pricing model, where the filtration $\left\{\mathcal{F}_{t}\right\}_{t \geq 0}$ represents the information resolved over time that is known to both firms. Since the optimal decision of investment is based on the current information $\mathcal{F}_{t}$, the optimal investment time is $\mathcal{F}_{t^{-}}$ measurable so that it is a stopping time. The value function is obtained by maximizing the expected revenue flow over all possible strategies (or stopping times). Further, the firms are assumed to be risk neutral or the risk factor has zero correlation with the market risk so that discount on value can be taken at the riskless interest rate.

\subsection{Follower value function}

We consider the optimal investment strategy of Firm $i$ which invests strictly later than its competitor Firm $i^{\prime}$. The follower value function $F_{i}(\theta)$ consists of two parts, $F_{i}^{(1)}(\theta)$ when Firm $i$ is still waiting for optimal entry and $F_{i}^{(2)}(\theta)$ when Firm $i$ has invested. Let $\theta_{i f}^{*}$ denote the investment threshold for optimal entry as a follower for Firm $i$ and $t_{i f}^{*}$ denote the optimal time of investment (stopping time). The optimal stopping time is given by the infimum of the time that $\theta$ reaches $\theta_{i f}^{*}$. When $\theta<\theta_{i f}^{*}$, the follower still holds an option to invest and revenue flow is received only after $t_{i f}^{*}$. The value function is given 
by

$$
F_{i}^{(1)}(\theta)=\max _{t_{i f}^{*}} E_{t}\left[\int_{t_{i f}^{*}}^{\infty} e^{-r(u-t)} D_{i d} \theta_{u} d u-e^{-r\left(t_{i f}^{*}-t\right)} K_{i}\right], \quad \theta<\theta_{i f}^{*},
$$

where $E_{t}$ denotes the expectation conditional on information available at time $t$ and $\theta_{t}=\theta$. The optimal time of investment entry $t_{i f}^{*}$ is chosen within the set of stopping times. When $\theta \geq \theta_{i f}^{*}$, the follower invests immediately so that its value function is given by

$$
F_{i}^{(2)}(\theta)=E_{t}\left[\int_{t}^{\infty} e^{-r(u-t)} D_{i d} \theta_{u} d u-K_{i}\right], \quad \theta \geq \theta_{i f}^{*}
$$

The closed form solution to $F_{i}^{(1)}$ and $F_{i}^{(2)}$ are known to be (Huisman, 2001)

$$
F_{i}(\theta)=\left\{\begin{array}{ll}
F_{i}^{(1)}(\theta), & \theta<\theta_{i f}^{*} \\
F_{i}^{(2)}(\theta), & \theta \geq \theta_{i f}^{*}
\end{array}= \begin{cases}\frac{K_{i}}{\beta-1}\left(\frac{\theta}{\theta_{i f}^{*}}\right)^{\beta}, & \theta<\theta_{i f}^{*} \\
\frac{D_{i d}}{r-\mu} \theta-K_{i}, & \theta \geq \theta_{i f}^{*},\end{cases}\right.
$$

where the threshold of optimal entry as a follower is given by

$$
\theta_{i f}^{*}=\frac{\beta}{\beta-1}(r-\mu) \widetilde{K}_{i d}
$$

with the parameter $\beta$ defined by

$$
\beta=\frac{1}{2}\left[1-\frac{2 \mu}{\sigma^{2}}+\sqrt{\left(1-\frac{2 \mu}{\sigma^{2}}\right)^{2}+\frac{8 r}{\sigma^{2}}}\right], \quad \beta>1 .
$$

When $\sigma \rightarrow 0^{+}$, while keeping $r$ and $\mu$ fixed, we then have $\beta \rightarrow \infty$ so that $\theta_{i f}^{*} \rightarrow(r-\mu) \widetilde{K}_{i d}$. Let $M_{i f}^{*}$ denote the Marshallian threshold value corresponding to the "net present value" rule. We observe that the limiting value of $\theta_{i f}^{*}$ at vanishing volatility is simply $M_{i f}^{*}$. This is expected since the Marshallian model corresponds to the zero volatility case. These threshold values are related by

$$
\theta_{i f}^{*}=\frac{\beta}{\beta-1} M_{i f}^{*}=\frac{\beta}{\beta-1}(r-\mu) \widetilde{K}_{i d}
$$




\subsection{Leader value functions}

Let $\theta_{i \ell}^{*}$ be the investment threshold for optimal entry as a leader for Firm $i$. Depending on the cost-to-revenue ratio $\widetilde{K}_{i j}$ in the duopoly and monopoly states of the two competing firms, one firm may or may not have comparative advantage over the other firm as the leader. When the competition is keen (whose detailed quantification is presented in Section 5), one of the two firms may choose pre-emptive entry. The pre-empting firm is called the pre-emptive leader. On the other hand, one of the two firms may be seen to be always better off by serving as the follower, its competitor can wait until the leader threshold is reached for optimal entry as a leader. In this case, the leader is called the dominant leader. We would like to derive the corresponding leader value function under both cases. The two leader value functions are equal when $\theta>\theta_{i \ell}^{*}$ since Firm $i$ would invest immediately.

Pre-emptive leader value function

When Firm $i$ is the pre-emptive leader, we are only interested to find the leader value function at the moment $t_{i p}$ at which Firm $i$ takes the pre-emptive action. Note that the revenue flow after entry assumes the value $D_{i m} \theta_{t}$ or $D_{i d} \theta_{t}$, for Firm $i$ as the first or second entrant, respectively. Assume $\theta$ to be sufficiently low, Firm $i$ 's pre-emptive leader function for $\theta<\theta_{i^{\prime} f}^{*}$ is given by

$$
\begin{aligned}
L_{i}^{(p)}(\theta)= & E_{t_{i p}}\left[\int_{t_{i p}^{*}}^{t_{i^{\prime} f}^{*}} e^{-r\left(u-t_{i p}^{*}\right)} D_{i m} \theta_{u} d u\right. \\
& \left.+\int_{t_{i^{\prime} f}^{*}}^{\infty} e^{-r\left(u-t_{i^{\prime} f}^{*}\right)} D_{i d} \theta_{u} d u-K_{i}\right]
\end{aligned}
$$

where $t_{i p}^{*}$ is the pre-emptive stopping time for Firm $i$. When $\theta \geq \theta_{i^{\prime} f}^{*}, L_{i}^{(p)}(\theta)$ can be obtained in a similar manner as $F_{i}^{(2)}(\theta)$ [see Eq. (3.2)]. The preemptive leader value function at $t_{i p}$ over the whole range of $\theta$ is found to be

$$
L_{i}^{(p)}(\theta)= \begin{cases}\frac{D_{i d}-D_{i m}}{r-\mu} \theta_{i^{\prime} f}^{*}\left(\frac{\theta}{\theta_{i^{\prime} f}^{*}}\right)^{\beta}+\frac{D_{i m}}{r-\mu} \theta-K_{i}, & \theta<\theta_{i^{\prime} f}^{*}, \\ \frac{D_{i d}}{r-\mu} \theta-K_{i}, & \theta \geq \theta_{i^{\prime} f}^{*} .\end{cases}
$$


Note that when $\theta \geq \theta_{i^{\prime} f}^{*}, L_{i}^{(p)}$ equals $F_{i}^{(2)}$. This is because once the rival has entered, returns to both firms are unchanged.

One may be puzzled by the possibility of negative value taken by $L_{i}^{(p)}(\theta)$ when $\theta$ assumes sufficiently low value. It will be shown later that pre-emptive action only occurs when the pre-emptive leader value function rises above the follower value function. Strictly speaking, $L_{i}^{(p)}(\theta)$ is defined only for those values of $\theta$ such that $L_{i}^{(p)}(\theta)>F_{i}(\theta)$. Since $F_{i}(\theta)$ is always positive, so $L_{i}^{(p)}(\theta)$ is guaranteed to be positive.

\section{Dominant leader value function}

Suppose Firm $i$ is the dominant leader so that he can wait until $\theta_{t}$ reaches the threshold $\theta_{i l}^{*}$ for optimal entry. The maximization procedure also takes into account the optimal entry made by the follower. Let $t_{i l}^{*}$ be the stopping time for optimal investment entry of the dominant leader and $L_{i}^{(d)}(\theta)$ denote the dominant leader value function. Since Firm $i$ as the leader is supposed to invest earlier than the follower Firm $i^{\prime}$, so $t_{i \ell}^{*}<t_{i^{\prime} f}^{*}$. For $t_{i \ell}^{*}<t_{i^{\prime} f}^{*}$ and $\theta<\theta_{i l}^{*}$, the value function $L_{i}^{(d)}(\theta)$ is given by

$$
\begin{gathered}
L_{i}^{(d)}(\theta)=\max _{t_{i l}^{*}} E_{t}\left[\int_{t_{i l}^{*}}^{t_{i^{\prime} f}^{*}} e^{-r(u-t)} D_{i m} \theta_{u} d u-e^{-r\left(t_{i l}^{*}-t\right)} K_{i}\right. \\
\left.+\int_{t_{i^{\prime} f}^{*}}^{\infty} e^{-r(u-t)} D_{i d} \theta_{u} d u\right] \\
=\frac{K_{i}}{\beta-1}\left(\frac{\theta}{\theta_{i l}^{*}}\right)^{\beta}+\frac{D_{i d}-D_{i m}}{r-\mu} \theta_{i^{\prime} f}^{*}\left(\frac{\theta}{\theta_{i^{\prime} f}^{*}}\right)^{\beta}, \quad \theta<\theta_{i \ell}^{*} .
\end{gathered}
$$

Here, leader's entry threshold of Firm $i$ is given by

$$
\theta_{i l}^{*}=\frac{\beta}{\beta-1}(r-\mu) \widetilde{K}_{i m} .
$$

It is observed that

$$
t_{i \ell}^{*}<t_{i^{\prime} f}^{*} \quad \Leftrightarrow \quad \theta_{i \ell}^{*}<\theta_{i^{\prime} f}^{*} \quad \Leftrightarrow \quad \widetilde{K}_{i m}<\widetilde{K}_{i^{\prime} d}
$$

When $\theta \geq \theta_{i l}^{*}$, Firm $i$ should invest immediately and so the dominant leader value function and its pre-emptive counterpart should have the same value. 
For $\theta \geq \theta_{i \ell}^{*}, L_{i}^{(d)}(\theta)$ is related to $L_{i}^{(p)}(\theta)$ and $F_{i}^{(2)}(\theta)$ as follows:

$$
L_{i}^{(d)}(\theta)=\left\{\begin{array}{ll}
L_{i}^{(p)}(\theta) & \theta_{i \ell}^{*} \leq \theta<\theta_{i^{\prime} f}^{*} \\
F_{i}^{(2)}(\theta) & \theta \geq \theta_{i^{\prime} f}^{*}
\end{array} .\right.
$$

\section{Pre-emptive thresholds}

In order for a firm to have the incentive to pre-empt its rival, its pre-emptive leader value must be larger than the follower value for a range of $\theta$, otherwise it is better off as follower. In this paper, it is always assumed that we start with sufficiently low value of $\theta$ so that both firms have to wait for their respective entry conditions to be met. Pre-emptive action taken by Firm $i$ may occur only when the pre-emptive leader value function $L_{i}^{(p)}(\theta)$ first surpasses the follower value function $F_{i}(\theta)$. We define the pre-emptive threshold $\theta_{i p}^{*}$ by

$$
\theta_{i p}^{*}=\inf \left\{\theta: L_{i}^{(p)}(\theta)>F_{i}(\theta)\right\} .
$$

According to the above definition, $\theta_{i p}^{*}$ does not exist when $L_{i}^{(p)}(\theta) \leq F_{i}(\theta)$ for all values of $\theta$. Since the two functions $L_{i}^{(p)}(\theta)$ and $F_{i}(\theta)$ are continuous, it follows that $L_{i}^{(p)}\left(\theta_{i p}^{*}\right)=F_{i}\left(\theta_{i p}^{*}\right)$. We call $\theta_{i p}^{*}$ the pre-emptive threshold, at which Firm $i$ is indifferent between leading by pre-emption and following.

The pre-emptive incentive can be quantified as the excess of the preemptive leader value over the follower value normalized by the sunk cost. Accordingly, we define the function

$$
\phi_{i}(\theta)=\frac{L_{i}^{(p)}(\theta)-F_{i}(\theta)}{K_{i}}, \quad i=1,2,
$$

as a measurement of pre-emptive incentive of Firm $i$. As seen from Eq. (3.6), $L_{i}^{(p)}(\theta)$ has different functional representations over the two domains: $\left[0, \theta_{i^{\prime} f}^{*}\right)$ and $\left[\theta_{i^{\prime} f}^{*}, \infty\right)$. Since pre-emption must occur before either firm's follower entry threshold is reached, we are only interested in $\phi_{i}(\theta)$ over the domain $\left[0, \min \left(\theta_{i f}^{*}, \theta_{i^{\prime} f}^{*}\right)\right)$. From the results in Eqs. (3.3) and (3.6), we obtain

$$
\phi_{i}(\theta)=-a_{i} \theta^{\beta}+b_{i} \theta-1, \quad \theta<\min \left(\theta_{i f}^{*}, \theta_{i^{\prime} f}^{*}\right), \quad i=1,2,
$$

where the coefficients are given by

$$
a_{i}=\frac{1}{\beta-1}\left[\beta\left(\frac{1}{\theta_{i \ell}^{*}}-\frac{1}{\theta_{i f}^{*}}\right)\left(\frac{1}{\theta_{i^{\prime} f}^{*}}\right)^{\beta-1}+\left(\frac{1}{\theta_{i f}^{*}}\right)^{\beta}\right]
$$




$$
b_{i}=\frac{\beta}{\beta-1} \frac{1}{\theta_{i \ell}^{*}}>0 .
$$

As a result, Eq. (4.1) is equivalent to

$$
\theta_{i p}^{*}=\inf \left\{\theta \in\left[0, \min \left(\theta_{i f}^{*}, \theta_{i^{\prime} f}^{*}\right)\right]: \phi_{i}(\theta)>0\right\} .
$$

By virture of continuity of $\phi_{i}$, we have $\phi_{i}\left(\theta_{i p}^{*}\right)=0$ and indeed $\theta_{i p}^{*}$ is the smallest positive root of $\phi_{i}$.

Furthermore, we define the following function that is relevant to our subsequent discussion of strategic interaction:

$$
q(x)=\left(\frac{1}{\beta} \frac{x^{\beta}-1}{x-1}\right)^{\frac{1}{\beta-1}} .
$$

Under the scenario of positive externalities where revenue flows are higher in duopoly state, firms should have no incentive be the leader. One would expect that $\theta_{i p}^{*}$ does not exist. The non-existence of $\theta_{i p}^{*}$ under positive externalities can be established mathematically by showing $L_{i}^{(p)}(\theta) \leq F_{i}(\theta)$, for all $\theta$. The details are presented in Appendix A. In our subsequent analysis of the mathematical properties of the pre-emptive thresholds, our discussion is then confined to the scenario of negative externalities.

Under negative externalities, it is obvious that $\theta_{i \ell}^{*}<\theta_{i f}^{*}$. Provided that $\theta_{i p}^{*}$ exists, it is relatively straightforward to establish $\theta_{i p}^{*}<\theta_{i \ell}^{*}$. We prove by contradiction by supposing $\theta_{i \ell}^{*} \leq \theta_{i p}^{*}$, then

$$
L_{i}^{(d)}\left(\theta_{i \ell}^{*}\right)=L_{i}^{(p)}\left(\theta_{i \ell}^{*}\right) \leq F_{i}\left(\theta_{i \ell}^{*}\right)
$$

The last inequality is due to the property: $L_{i}^{(p)}(\theta) \leq F_{i}(\theta)$ for any $\theta \leq$ $\theta_{i p}^{*}$, by virtue of definition (4.1). However, under negative externalities, the dominant leader value must be strictly larger than the follower value at $\theta_{i \ell}^{*}$ [for details, see Huisman's (2001) text, Sec. 4.2]. A contradiction is encountered, and so $\theta_{i p}^{*}<\theta_{i \ell}^{*}$. Therefore, the pre-emptive threshold, leader's and follower's entry thresholds observe the following order of relative magnitude.

$$
\theta_{i p}^{*}<\theta_{i \ell}^{*}<\theta_{i f}^{*}
$$

For Firm $i$, given its sunk cost and revenue flows in monopoly and duopoly states, its follower threshold $\theta_{i f}^{*}$ and leader threshold $\theta_{i \ell}^{*}$ can be determined. 
Suppose we define $\bar{R}_{i}=\frac{\theta_{i f}^{*}}{\theta_{i \ell}^{*}}=\frac{\widetilde{K}_{i d}}{\widetilde{K}_{i m}}$, then $\bar{R}_{i}>1(<1)$ under negative (positive) externalities. The special case $\bar{R}_{i}=1$ is ruled out since we assume either $D_{i m}>D_{i d}$ or $D_{i m}<D_{i d}$. The parameter $\bar{R}_{i}$ measures Firm $i$ 's first mover incentive, where lower $\bar{R}_{i}$ means higher incentive. The extent that Firm $i^{\prime}$ influences the strategic entry decision of Firm $i$ would depend on their follower threshold ratio $R_{i f}=\frac{\theta_{i f}^{*}}{\theta_{i^{\prime} f}^{*}}=\frac{\widetilde{K}_{i d}}{\widetilde{K}_{i^{\prime} d}}$ and leader threshold ratio $R_{i \ell}=\frac{\theta_{i \ell}^{*}}{\theta_{i^{\prime} \ell}^{*}}=\frac{\widetilde{K}_{i m}}{\widetilde{K}_{i^{\prime} m}}$. Taking Firm $i$ as the reference firm, with known values of $\widetilde{K}_{i d}$ and $\widetilde{K}_{i m}, \bar{R}_{i}$ can be considered fixed. It will be shown later that $R_{i f}$ and $R_{i \ell}$ are the two key parameters in the characterization of various forms of strategic equilibriums. In economic sense, $R_{i f}$ measures the competitive advantage of Firm $i$ as follower while $R_{i \ell}$ quantifies its competitive advantage as leader. The smaller $R_{i f}$, the greater Firm $i$ 's follower strength. Similarly, Firm $i$ 's leader strength is inversely related to $R_{i \ell}$.

The analysis of strategic equilibriums under negative externalities depend on the existence of the pre-emptive thresholds of the two rival firms and their relative magnitude. These properties are summarized in the following two propositions. In Figure 1, we plot $\phi_{i}(\theta)$ against $\theta$ for varying values of $R_{i f}$. The existence of $\theta_{i p}^{*}$ is seen to be related to the dependence of $\phi_{i}(\theta)$ on the parameter $R_{i f}$. The precise conditions under which the pre-emptive threshold exists are stated in Proposition 1. Proposition 2 determines which firm has a lower pre-emptive threshold. The corresponding criteria are derived from the analysis of the interaction of the two functions, $\phi_{i}(\theta)$ and $\phi_{i^{\prime}}(\theta)$.

\section{Proposition 1}

Under negative externalities, the pre-emptive threshold $\theta_{i p}^{*}$ of Firm $i$ exists if and only if $0<R_{i f}<q\left(\bar{R}_{i}\right)$, where $q\left(\bar{R}_{i}\right)>1$.

\section{Corollaries}

1. The pre-emptive thresholds of both firms exist if and only if

$$
\frac{1}{q\left(\bar{R}_{i^{\prime}}\right)}<R_{i f}<q\left(\bar{R}_{i}\right)
$$

where $\bar{R}_{i^{\prime}}=\theta_{i^{\prime} f}^{*} / \theta_{i^{\prime} \ell}^{*}$. 
2. When $R_{i f} \notin\left(\frac{1}{q\left(\bar{R}_{i^{\prime}}\right)}, q\left(\bar{R}_{i}\right)\right)$, only one pre-emptive threshold exists. Specifically, we have (i) for $0<R_{i f} \leq \frac{1}{q\left(\bar{R}_{i^{\prime}}\right)}, \theta_{i p}^{*}$ exists but not $\theta_{i^{\prime} p}^{*}$; and (ii) for $R_{i f} \geq q\left(\bar{R}_{i}\right), \theta_{i^{\prime} p}^{*}$ exists but not $\theta_{i p}^{*}$.

3. In the $R_{i f}-R_{i \ell}$ plane, the region where both pre-emptive thresholds exist is bounded by the following inequalities: $R_{i f}<q\left(\bar{R}_{i}\right), R_{i \ell}>\frac{R_{i f}}{\bar{R}_{i}}$ and $R_{i \ell}>\frac{R_{i f}}{\bar{R}_{i}} q^{-1}\left(\frac{1}{R_{i f}}\right)$.

The proofs of Proposition 1 and its corollaries are presented in Appendix B. Corollary 1 gives the conditions for the existence of both pre-emptive thresholds. Corollary 2 shows that at least one pre-emptive threshold exists under negative externalities. Furthermore, we can deduce that when the follower threshold of the rival firm is lower, the incentive for Firm $i$ to preempt the rival becomes smaller. From Corollary 2, part (ii), we deduce that when the rival's follower threshold $\theta_{i^{\prime} f}^{*}$ satisfies

$$
\theta_{i^{\prime} f}^{*} \geq \theta_{i f}^{*} / q\left(\frac{\theta_{i f}^{*}}{\theta_{i \ell}^{*}}\right)
$$

then Firm $i$ would never choose to pre-empt at all.

Recall that the two parameters $R_{i f}$ and $R_{i \ell}$ measure the follower and leader strengths of Firm $i$, respectively, with lower ratio represents greater strength. The full characterization of the existence properties of the preemptive thresholds can be analyzed in the parameter space of $R_{i f}$ and $R_{i \ell}$ (see Figure 2). Under the assumption of negative externalities, the parameters $\bar{R}_{i}$ and $\bar{R}_{i^{\prime}}$ are both greater than one. For a fixed value of the parameter $\bar{R}_{i}$, the requirement of $\bar{R}_{i^{\prime}}>1$ is equivalent to $R_{i \ell}>\frac{R_{i f}}{\bar{R}_{i}}$. Within the corresponding feasible region $\left\{\left(R_{i f}, R_{i \ell}\right): R_{i \ell}>\frac{R_{i f}}{\bar{R}_{i}}\right\}$ in the $R_{i f}-R_{i \ell}$ plane, Corollary 3 states that the region where both pre-emptive thresholds exist is given by the intersection of the region left of the vertical line: $R_{i f}=q\left(\bar{R}_{i}\right)$ and the region above the curve: $R_{i \ell}=\frac{R_{i f}}{\bar{R}_{i}} q^{-1}\left(\frac{1}{R_{i f}}\right)$. The latter curve intersects the oblique line: $R_{i \ell}=\frac{R_{i f}}{\bar{R}_{i}}$ at the point $\left(1, \frac{1}{\bar{R}_{i}}\right)$. 
When both $\theta_{i p}^{*}$ and $\theta_{i^{\prime} p}^{*}$ exist, we are interested to determine their relative magnitude. In Figure 2, the two regions in the $R_{i f}-R_{i \ell}$ plane

$$
\left\{\left(R_{i f}, R_{i \ell}\right): \theta_{i p}^{*}>\theta_{i^{\prime} p}^{*}\right\} \quad \text { and } \quad\left\{\left(R_{i f}, R_{i \ell}\right): \theta_{i p}^{*}<\theta_{i^{\prime} p}^{*}\right\}
$$

are separated by the curve: $\left\{\left(R_{i f}, R_{i \ell}\right): \theta_{i p}^{*}=\theta_{i^{\prime} p}^{*}\right\}$. This separating curve is seen to pass through the point $(1,1)$, which corresponds to the case of symmetric firms. It also intersects tangentially the vertical boundary line: $R_{i f}=q\left(\bar{R}_{i}\right)$ and the boundary curve: $R_{i \ell}=\frac{R_{i f}}{\bar{R}_{i}} q^{-1}\left(\frac{1}{R_{i f}}\right)$. Note that the curve is not symmetric with respect to the point $(1,1)$.

Let us first consider the characterization of the separating curve in the region $\left\{\left(R_{i f}, R_{i \ell}\right): R_{i f}<1\right.$ and $\left.R_{i \ell}>1\right\}$. This corresponds to the scenario of so-called "keen competition", where Firm $i$ has comparative advantage only as a follower but not as a leader. When $R_{i f}<1$ and $R_{i \ell}>1$, it can be shown that the two functions $\phi_{i}(\theta)$ and $\phi_{i^{\prime}}(\theta)$ is guaranteed to intersect at unique point

$$
\widehat{\theta}=\left(\frac{b_{i^{\prime}}-b_{i}}{a_{i^{\prime}}-a_{i}}\right)^{\frac{1}{\beta-1}}>0 .
$$

Here, $a_{i}, a_{i^{\prime}}, b_{i}$ and $b_{i^{\prime}}$ are defined in Eqs. (4.4a,b). As shown in Proposition 2 below, the relative magnitude of the pre-emptive thresholds depends on the sign behaviors of the following two quantities: $\phi_{i}(\widehat{\theta})$ and $\frac{d \phi_{i^{\prime}}}{d \theta}(\widehat{\theta})$.

\section{Proposition 2}

Negative externalities and existence of both pre-emptive thresholds $\theta_{i p}^{*}$ and $\theta_{i^{\prime} p}^{*}$ are assumed. Under the scenario of $R_{i f}<1$ and $R_{i \ell}>1$, the pre-emptive thresholds exhibit the following properties on their relative magnitude:

1. $\phi_{i}(\widehat{\theta})>0 \Rightarrow \theta_{i p}^{*}>\theta_{i^{\prime} p}^{*}$

2. $\phi_{i}(\widehat{\theta})=0$
(a) $\frac{d \phi_{i^{\prime}}}{d \theta}(\widehat{\theta})>0 \Rightarrow \theta_{i p}^{*}=\theta_{i^{\prime} p}^{*}$
(b) $\frac{d \phi_{i^{\prime}}}{d \theta}(\widehat{\theta})<0 \Rightarrow \theta_{i p}^{*}>\theta_{i^{\prime} p}^{*}$

3. $\phi_{i}(\widehat{\theta})<0$ 

(a) $\frac{d \phi_{i^{\prime}}}{d \theta}(\widehat{\theta})>0 \quad \Rightarrow \quad \theta_{i p}^{*}<\theta_{i^{\prime} p}^{*}$
(b) $\frac{d \phi_{i^{\prime}}}{d \theta}(\widehat{\theta})<0 \Rightarrow \theta_{i p}^{*}>\theta_{i^{\prime} p}^{*}$.

The proof of Proposition 2 is presented in Appendix C. Let $P$ denote the point of intersection of $\phi_{i}(\theta)$ and $\phi_{i^{\prime}}(\theta)$. The results in Proposition 2 are related to whether the position of $P$ is staying above or below the $\theta$-axis and whether $P$ lies on the increasing part or decreasing part of the curve $\phi_{i^{\prime}}(\theta)$ [see Figures 3a-3e for details]. Provided that the values of the leader and follower thresholds of both firms are known, $\phi_{i}(\widehat{\theta})$ and $\frac{d \phi_{i^{\prime}}}{d \theta}(\widehat{\theta})$ can be readily computed. The criteria stated in Proposition 2 can then be checked easily to determine the relative magnitude of $\theta_{i p}^{*}$ and $\theta_{i^{\prime} p}^{*}$.

In Figure 2, the part of the separating curve corresponding to $\theta_{i p}^{*}=\theta_{i^{\prime} p}^{*}$ that lies inside the region $\left\{\left(R_{i f}, R_{i \ell}\right): R_{i f}<1\right.$ and $\left.R_{i \ell}>1\right\}$ is given by the part of the curve defined by $\phi_{i}(\widehat{\theta})=0$ satisfying $\frac{d \phi_{i^{\prime}}}{d \theta}(\widehat{\theta})>0$ [see Part 2 (a) in Proposition 2]. As for theoretical interest, the part of the curve: $\phi_{i}(\widehat{\theta})=0$ satisfying $\frac{d \phi_{i^{\prime}}}{d \theta}(\widehat{\theta})<0$ lies completely inside the region $\theta_{i p}^{*}>\theta_{i^{\prime} p}^{*}$, which is in agreement with Part 2(b) in Proposition 2.

We then consider the scenario where $R_{i \ell}>1$ and $R_{i f} \geq 1$. In this case, Firm $i^{\prime}$ has competitive advantage over Firm $i$ in monopoly state and no less advantage in duopoly state. It can be shown easily that $\phi_{i^{\prime}}(\theta)>\phi_{i}(\theta)$ for all values of $\theta \in\left(0, \min \left(\theta_{i f}^{*}, \theta_{i^{\prime} f}^{*}\right)\right)$. Accordingly, we deduce that (i) $\theta_{i^{\prime} p}^{*}<\theta_{i p}^{*}$ if both $\theta_{i p}^{*}$ and $\theta_{i^{\prime} p}^{*}$ exists or (ii) only $\theta_{i^{\prime} p}^{*}$ exists. In Figure 2, we observe that when $R_{i f} \geq q\left(\bar{R}_{i}\right)>1$, then $\theta_{i p}^{*}$ does not exist, a result that is in agreement with Corollary 2(ii) of Proposition 1.

Next, we consider the two special cases (i) $R_{i \ell}=1$ and $R_{i f}<1$ and (ii) $R_{i \ell}=R_{i f}=1$. For case (i), it is seen that $\phi_{i}(\theta)>\phi_{i^{\prime}}(\theta)$ for $\theta>0$ so that $\theta_{i p}^{*}<\theta_{i^{\prime} p}^{*}$ if both $\theta_{i p}^{*}$ and $\theta_{i^{\prime} p}^{*}$ exists or else $\theta_{i^{\prime} p}^{*}$ does not exist at all (see Figure 2). The second case represents the interesting scenario of symmetry in both firms. When $R_{i \ell}=R_{i f}=1$, we have $\theta_{i \ell}^{*}=\theta_{i^{\prime} \ell}^{*}$ and $\theta_{i f}^{*}=\theta_{i^{\prime} f}^{*}$ so that $\phi_{i}(\theta)=\phi_{i^{\prime}}(\theta)$ and thus $\theta_{i p}^{*}=\theta_{i^{\prime} p}^{*}$. As shown in Figure 2, the separating curve: $\theta_{i p}^{*}=\theta_{i^{\prime} p}^{*}$ does pass through the point $(1,1)$ (labeled as a circle).

Since $\left\{R_{i f}>1, R_{i \ell} \leq 1\right\}$ is equivalent to $\left\{R_{i^{\prime} f}<1, R_{i^{\prime} \ell} \geq 1\right\}$, and similar equivalence for $\left\{R_{i f} \leq 1, R_{i \ell}<1\right\}$ and $\left\{R_{i^{\prime} f} \geq 1, R_{i^{\prime} \ell}>1\right\}$, the properties on the relative magnitude of $\theta_{i p}^{*}$ and $\theta_{i^{\prime} p}^{*}$ under the scenario of 
$\left\{R_{i f}>1, R_{i \ell} \leq 1\right\}$ and $\left\{R_{i f} \leq 1, R_{i \ell}<1\right\}$ can be deduced from the earlier results by interchanging the roles of $i$ and $i^{\prime}$. Hence, we have completed the characterization of the relative magnitude of the pre-emptive thresholds of the two firms in the whole $R_{i f}-R_{i \ell}$ plane.

The assumption of "asymmetry on cost only" in earlier papers [Huisman et al. (2004), Kijima and Shibata (2002)] would limit their consideration of strategic equilibriums to $R_{i \ell}<1$ and $R_{i f}<1$. This corresponds to the less interesting scenario of one firm having competitive advantage over its rival in both states. As evident from Fig. 2, cost asymmetry alone always yields leadership by the low-cost firm. Both Huisman et al. (2004) and Pawlina (2003) postulate that simultaneous entry occurs when $K \in\left(1, K^{* *}\right)$, where $K$ is the ratio of the high cost to the low cost and $K^{* *}=\max \left\{\frac{1}{q\left(\bar{R}_{i}\right)}, 1\right\}$. However, under negative externalities, we always have $q\left(\bar{R}_{i}\right)>1$. This leads to $K^{* *}=1$ so the low-cost firm always leads and there will be no simultaneous entry. Our analysis shows that simultaneous entry is triggered by coincidence of pre-emptive thresholds, $\theta_{i p}^{*}=\theta_{i^{\prime} p}^{*}$, which is different from the simple case of identical pre-emptive threshold in symmetric firms. Actually, our model assumption of "asymmetry on both cost and revenue" induces a complete characterization of strategic equilibriums and only under such assumption that it is possible to simulate all possible relative positions of rival's leader and follower threshold values. The ratios $\bar{R}_{i}, R_{i f}$ and $R_{i \ell}$ are the fundamental parameters that determine various forms of equilibrium. Investors should consider cost normalized by revenue when they decide on their investment strategy.

Under the assumption of negative externalities, we also investigate the impact of varying volatility values on the two regions: $\theta_{i p}^{*}<\theta_{i^{\prime} p}^{*}$ and $\theta_{i^{\prime} p}^{*}<\theta_{i p}^{*}$, and the pre-emptive thresholds. In Figure 4 , we plot the separating curve: $\theta_{i p}^{*}=\theta_{i^{\prime} p}^{*}$ with varying values of $\beta$ (high $\beta$ means lower volatility $\sigma$ ) in the $R_{i f^{-}}$ $R_{i \ell}$ plane. Consider the region $\left\{\left(R_{i f}, R_{i \ell}\right): R_{i f}<1\right.$ and $\left.R_{i \ell}>1\right\}$, an increase in volatility (lower value of $\beta$ ) steepens the separating curve upward about the pivot point $(1,1)$, at which $R_{i f}=R_{i \ell}=1$. Suppose we fix the value of $R_{i \ell}$ and gradually decrease the value of $R_{i f}$, we cross from the region: $\left\{\theta_{i p}^{*}>\theta_{i^{\prime} p}^{*}\right\}$ to the region: $\left\{\theta_{i p}^{*}<\theta_{i^{\prime} p}^{*}\right\}$ at a lower value of $R_{i f}$ under the scenario of higher value of $\beta$ (lower volatility).

In summary, the firm with greater follower strength will hasten preemption as the level of uncertainty increases, while its rival will delay en- 
try. To see the reason, suppose we assume that Firm $i$ has greater follower strength and $\theta_{i f}<\theta_{i^{\prime} f}$. When $\sigma$ increases, both firms' follower thresholds and follower value functions increase. However, the increase in follower thresholds would increase the pre-emptive leader value functions by making the first term of Eq. (3.6) less negative. To Firm $i$, its increase in leader value would outweigh its follower value to a greater extent than its rival. To its rival, the increase in follower value outweights the gain in pre-emptive leader value, so it prefers to delay. This agrees with the economic intuition that at a lower level of volatility, it would require a stronger competitive advantage in duopoly state (smaller value of $R_{i f}$ ) to enhance the pre-emptive incentive. From Figure $5, \theta_{i p}^{*}\left(\theta_{i^{\prime} p}^{*}\right)$ is seen to be decreasing (increasing) with increasing volatility level when $R_{i f}<1$ and $R_{i \ell}>1$. With higher volatility, the pre-emptive incentive of Firm $i$ (Firm $i^{\prime}$ ) becomes greater (less) so that pre-emptive action becomes more (less) favorable, thus leading to a lower (higher) value of pre-emptive threshold.

\section{$5 \quad$ Strategic equilibriums}

In this section, we analyze the strategic investment decisions of the two competing firms under various forms of strategic equilibriums. The different outcomes of investment decisions depend on the existence properties of the pre-emptive thresholds. These properties are summarized below:

1. Under positive externalities, both firms do not have the incentive to pre-emptive so that $\theta_{i p}^{*}$ and $\theta_{i^{\prime} p}^{*}$ do not exist.

2. Under negative externalities, it may occur that

a. only one of the pre-emptive thresholds exists;

b. both pre-emptive thresholds exist and one is strictly smaller than the other;

c. the two pre-emptive thresholds are equal.

The different cases of existence of the pre-emptive thresholds would lead to various forms of strategic equilibrium, which may be sequential entry equilibrium, simultaneous entry equilibrium or pre-emptive entry equilibrium. 


\subsection{Positive externalities}

Under positive externalities, there are no pre-emptive thresholds and the leader and follower thresholds observe

$$
\theta_{i f}^{*}<\theta_{i \ell}^{*} \quad \text { and } \quad \theta_{i^{\prime} f}^{*}<\theta_{i^{\prime} \ell}^{*} .
$$

Without loss of generality, we may assume $\theta_{i f}^{*} \leq \theta_{i^{\prime} f}^{*}$, corresponding to $R_{i f} \leq$ 1. There are three possible orderings of the thresholds

(i) $\theta_{i f}^{*} \leq \theta_{i^{\prime} f}^{*}<\theta_{i^{\prime} \ell}^{*} \leq \theta_{i \ell}^{*}$

(ii) $\theta_{i f}^{*} \leq \theta_{i^{\prime} f}^{*} \leq \theta_{i \ell}^{*} \leq \theta_{i^{\prime} \ell}^{*}$

(iii) $\theta_{i f}^{*}<\theta_{i \ell}^{*} \leq \theta_{i^{\prime} f}^{*} \leq \theta_{i^{\prime} \ell}^{*}$.

The form of strategic equilibrium depends on whether the leader threshold $\theta_{i \ell}^{*}$ of Firm $i$ comes after or before the follower threshold $\theta_{i^{\prime} f}^{*}$ of its rival, that is, $\theta_{i \ell}^{*} \geq \theta_{i^{\prime} f}^{*}$ or $\theta_{i \ell}^{*} \leq \theta_{i^{\prime} f}^{*}$.

First, we consider $\theta_{i \ell}^{*} \geq \theta_{i^{\prime} f}^{*}$. In this case, both firms would invest simultaneously at $\theta_{i^{\prime} f}^{*}$, resulting in simultaneous entry equilibrium. Knowing that Firm $i^{\prime}$ would invest optimally as a follower when $\theta \geq \theta_{i^{\prime} f}^{*}$, Firm $i$ would invest at $\theta_{i^{\prime} f}^{*}$ since the follower value of Firm $i$ at $\theta=\theta_{i^{\prime} f}^{*}$ is higher than its leader value due to positive externalities. For the second case where $\theta_{i \ell}^{*} \leq \theta_{i^{\prime} f}^{*}$, Firm $i$ would invest at $\theta_{i \ell}^{*}$. This is because at $\theta=\theta_{i \ell}^{*}$ it is optimal for Firm $i$ to enter as a leader. Firm $i^{\prime}$ would enter later at $\theta_{i^{\prime} f}^{*}$ as a follower. This results in sequential entry equilibrium. In summary, unless the optimal stopping time of leader entry for Firm $i$ comes before that of the follower entry for its rival, Firm $i$ prefers to wait until the stopping time for simultaneous entry of both firms.

\subsection{Negative externalities}

First, we consider the case where Firm $i$ dominates in the sense that only $\theta_{i p}^{*}$ exists but not $\theta_{i^{\prime} p}^{*}$. Here, Firm $i^{\prime}$ would never take pre-emptive action since its pre-emptive leader value is always lower than its follower value. Hence, Firm $i$ can choose its optimal entry strategy without the influence of its rival. Under negative externalities, we have $\theta_{i \ell}^{*}<\theta_{i f}^{*}$ so that Firm $i$ chooses to invest at $\theta=\theta_{i \ell}^{*}$ as optimal leader entry. Subsequently, Firm $i^{\prime}$ will invest optimally at a later time when $\theta$ reaches $\theta_{i^{\prime} f}^{*}$. This results in sequential entry equilibrium. 
Next, we consider the scenario of "keen competition" where the preemptive threshold of both firms exist. In Proposition 2, we present the criteria to determine which pre-emptive threshold has a lower value or whether both threshold values are equal. Without loss of generality, let us assume $\theta_{i p}^{*}<\theta_{i^{\prime} p}^{*}$, which infers that Firm $i$ has stronger pre-emptive advantage. Firm $i$ does not have to take pre-emptive action to invest at $\theta_{i p}^{*}$ as Firm $i^{\prime}$ will refrain from entry when

$$
L_{i^{\prime}}^{(p)}(\theta)<F_{i^{\prime}}(\theta) \text { for } \theta<\theta_{i^{\prime} p}^{*}
$$

The optimal strategy for Firm $i$ is to invest at $\min \left(\theta_{i \ell}^{*}, \theta_{i^{\prime} p}^{*}\right)$. More precisely, when $\theta_{i \ell}^{*} \leq \theta_{i^{\prime} p}^{*}$, Firm $i$ will invest at $\theta_{i \ell}^{*}$ as optimal leader entry. Otherwise, when $\theta_{i \ell}^{*}>\theta_{i^{\prime} p}^{*}$, Firm $i$ enters at $\theta_{i^{\prime} p}^{*}$ as pre-emptive entry. The relative magnitude of $\theta_{i \ell}^{*}$ and $\theta_{i^{\prime} p}^{*}$ under keen competition is stated in Proposition 3.

\section{Proposition 3}

Under negative externalities and assuming both $\theta_{i p}$ and $\theta_{i^{\prime} p}$ exist, we have

$$
\begin{aligned}
& \theta_{i \ell}^{*} \leq \theta_{i^{\prime} p}^{*} \\
\Leftrightarrow & \beta \bar{R}_{i}^{\beta}\left(R_{i \ell}-1\right) \leq \beta\left(\bar{R}_{i} R_{i \ell}-R_{i f}\right)+R_{i f}^{\beta}<\bar{R}_{i}^{\beta} .
\end{aligned}
$$

The proof of Proposition 3 is presented in Appendix D. Actually, the potential pre-emptive action of Firm $i^{\prime}$ has no effect on the strategic investment decision of Firm $i$ when $\theta_{i \ell}^{*} \leq \theta_{i^{\prime} p}^{*}$. This equilibrium outcome is indistinguishable from the dominant case when $\theta_{i p}$ alone exists. Again, we have sequential entry equilibrium since Firm $i^{\prime}$ will invest at $\theta=\theta_{i^{\prime} f}^{*}$ at a later time.

When $\theta_{i \ell}^{*}>\theta_{i^{\prime} p}^{*}$, it is obvious that Firm $i$ will not wait till $\theta_{i \ell}^{*}$ is reached before entry, for its rival can pre-empt at $\theta_{i^{\prime} p}^{*}$. Between $\theta_{i p}^{*}$ and $\theta_{i^{\prime} p}^{*}$, Firm $i$ will choose to delay entry till as close to $\theta_{i^{\prime} p}^{*}$ as possible. This strategy not only yields greatest exercise value on Firm $i$ 's own leader option among all $\theta \in\left[\theta_{i p}^{*}, \theta_{i^{\prime} p}^{*}\right)$, it also guarantees Firm $i$ leadership over its rival. Being aware of Firm $i$ 's strategy, Firm $i^{\prime}$ will choose to invest at $\theta_{i^{\prime} f}^{*}$. Effectively, Firm $i$ will invest at $\theta_{i^{\prime} p}^{*}$ and Firm $i^{\prime}$ at $\theta_{i^{\prime} f}^{*}$, results in pre-emptive entry equilibrium.

Under the special case where $\theta_{i p}^{*}=\theta_{i^{\prime} p}^{*}$, by following similar argument as above, we also have simultaneous pre-emptive entry of both firms at $\theta_{i p}^{*}$, except when the two firms are symmetric. Under symmetry of the two firms, each firm will have $50 \%$ chance of successfully pre-empting its rival (Fudenberg and Tirole, 1985). 


\section{Conclusion}

Our work combines two streams of research in investment science: investment under competition and investment under uncertainty. We analyze various forms of strategic equilibriums in investment games between two firms that compete for entry in a project that generates uncertain revenue flows. Under the assumption of asymmetry in both costs and revenue flows, our model provides a wider set of strategic interaction of entry decisions. In particular, we demonstrate that when no firm enjoys competitive advantage in both monopoly and duopoly states, it is possible to yield simultaneous entry. This cannot happen under the framework of asymmetry in cost alone, since in such scenario the lower cost firm would have competitive advantage over its rival throughout, thus resulting in either sequential or pre-emptive entry equilibrium.

We have derived the value functions for entry as a follower, pre-emptive leader or dominant leader. In particular, we have provided a complete characterization of strategic games between two competing firms facing single entry decision. We have shown that when analysis is performed in the $R_{i f}$ - $R_{i \ell}$ parameter space, various forms of entry equilibriums can be clearly presented. The set of strategic equilibriums include pre-emptive entry equilibrium, sequential entry equilibrium and simultaneous entry equilibrium.

Under positive externalities, both firms do not have the incentive to preempt. If the leader's threshold of one firm is higher than the follower's threshold of its rival, then both firms would invest simultaneously at the higher of the two follower's thresholds. Otherwise, sequential entry equilibrium is resulted where the firm with lower leader's threshold would choose optimal entry as a leader while its rival would enter later at the optimal followers threshold as a follower.

Under the scenario of negative externalities, when one firm dominates the other, we again have sequential entry equilibrium. Dominance occurs when either the pre-emptive threshold of only one firm exists or the leader's threshold of one firm is lower than the pre-emptive threshold of its rival. Without dominance, the game equilibriums can be either pre-emptive or simultaneous. In either case, the threat of pre-emption by rival leads to pre-emptive entry or simultaneous entry.

We also examine the influence of market uncertainty as measured by the volatility of the underlying stochastic state variable on the pre-emptive entry equilibriums. In the strategic investment game between two firms with 
asymmetry in both cost and revenue, we find that an increase in volatility may hasten or delay pre-emptive entry. Our numerical example shows that increasing volatility considerably hastens pre-emptive entry by the firm with greater follower strength.

\section{References}

[1] Boyer, M., E. Gravel, P. Lasserre. 2004. Real options and strategic competition: a survey. Working paper of Univ. de Montreal.

[2] Dixit, A., R. Pindyck. 1994. Investment under Uncertainty, Princeton University Press, Princeton.

[3] Fudenberg, D., J. Tirole. 1985. Preemption and rent equalization in the adoption of new technology. Review of Economic Studies 52, 383-401.

[4] Grenadier, S. 1996. The strategic exercise of options: Development cascades and overbuilding in real estate markets. Journal of Finance 51, 1653-1679.

[5] Huisman, K. 2001. Technology investment: A game theoretic real options approach, Kluwer Academic Publishers.

[6] Huisman, K., P. Kort, G. Pawlina, J. Thijssen. 2004. Strategic investment under uncertainty: Merging real options with game theory. Zeitschrift fur Betriebswirtschaft 67, 97-123.

[7] Joaquin, D., K. Butler. 1999. Competitive investment decisions: A synthesis, in Project flexibility, agency and competition, edited by M. Brennan and L. Trigeorgis.

[8] Kijima, M., T. Shibata. 2002. Real options in a duopoly market with general volatility structure. Working paper of Kyoto University.

[9] Mason, R., H. Weeds. 2005. Can greater uncertainty hasten investment? Working paper of University of Southhampton and University of Essex.

[10] Pawlina, G., P. Kort. 2006. Real options in an asymmetric duopoly: Who benefits from your competition. Journal of Economics and Management Strategy 15, 1-35. 
[11] Pawlina, G. 2003. Corporate investment under uncertainty and competition: A real options approach. PhD thesis, Tilburg University, the Netherlands. 


\section{Appendix A - Non-existence of pre-emptive threshold under pos-} itive externalities

We would like to show that $\phi_{i}(\theta) \leq 0$ for $\theta \in\left(0, \min \left(\theta_{i f}^{*}, \theta_{i^{\prime} f}^{*}\right)\right)$ under positive externalities. When $\theta<\theta_{i f}^{*}$, we have $F_{i}^{(2)}(\theta)<F_{i}^{(1)}(\theta)$. This is because the continuation value $F_{i}^{(1)}(\theta)$ should be higher than the exercise value $F_{i}^{(2)}(\theta)$ when $\theta$ has not reached the threshold $\theta_{i f}^{*}$. It suffices to show that

$$
L_{i}^{(p)}(\theta)-F_{i}^{(2)}(\theta) \leq 0 \text { for } \theta<\theta_{i^{\prime} f}^{*}
$$

We write $\theta=\alpha \theta_{i^{\prime} f}^{*}$, where $0 \leq \alpha<1$. It is seen that

$$
L_{i}^{(p)}(\theta)-F_{i}^{(2)}(\theta)=\frac{D_{i d}-D_{i m}}{r-\mu}\left(\alpha \theta_{i^{\prime} f}^{*}\right)\left(\alpha^{\beta-1}-1\right) .
$$

Since $D_{i d}>D_{i m}$ under positive externalities and $\alpha^{\beta-1}-1<0$, we obtain the required result.

\section{Appendix B - Proof of Proposition 1}

Under negative externalities, we have $\theta_{i \ell}^{*}<\theta_{i f}^{*}$ so that $a_{i}>0$ and $\bar{R}_{i}>1$. Knowing that $\beta$ is always greater than 1 , the function $\phi_{i}(\theta)$ is concave since

$$
\phi_{i}^{\prime \prime}(\theta)=-\beta(\beta-1) a_{i}<0 .
$$

To prove the existence of $\theta_{i p}^{*}$ when $R_{i f} \in\left(0, q\left(\bar{R}_{i}\right)\right)$, we consider the two separate cases (i) $0<R_{i f}<1$ and (ii) $R_{i f} \geq 1$.

(i) When $0<R_{i f}<1$, the domain of definition of $\phi_{i}(\theta)$ is $\left[0, \theta_{i f}^{*}\right]$. At the two end points $\theta=0$ and $\theta=\theta_{i f}^{*}$, the functional values of $\phi_{i}(\theta)$ are

$$
\phi_{i}(0)=-1 \quad \text { and } \quad \phi_{i}\left(\theta_{i f}^{*}\right)=\frac{\beta}{\beta-1}\left(\bar{R}_{i}-1\right)\left(1-R_{i f}\right)^{\beta-1}>0 .
$$

By the continuity and concavity properties of $\phi_{i}$, the function $\phi_{i}$ has unique root $\theta_{i p}^{*} \in\left(0, \theta_{i f}^{*}\right)$ (see the most upper curve in Figure 1$)$. 
(ii) When $R_{i f} \geq 1$, the domain of definition of $\phi_{i}(\theta)$ becomes $\left[0, \theta_{i^{\prime} f}^{*}\right]$. Considering

$$
\begin{aligned}
\phi_{i}\left(\theta_{i^{\prime} f}^{*}\right) & =\frac{1}{K_{i}}\left\{L_{k}^{(p)}\left(\theta_{i^{\prime} f}^{*}\right)-F_{i}^{(1)}\left(\theta_{i^{\prime} f}^{*}\right)\right\} \\
& =\frac{1}{K_{i}}\left\{\frac{D_{i d}}{r-\mu} \theta_{i^{\prime} f}^{*}-K_{i}-\frac{K_{i}}{\beta-1}\left(\frac{\theta_{i^{\prime} f}^{*}}{\theta_{i f}^{*}}\right)^{\beta}\right\} .
\end{aligned}
$$

Here, $\frac{D_{i d}}{r-\mu} \theta_{i^{\prime} f}^{*}-K_{i}$ is seen to be $F_{i}^{(2)}\left(\theta_{i^{\prime} f}^{*}\right)$. By observing that

$$
F_{i}^{(2)}(\theta) \leq F_{i}^{(1)}(\theta) \quad \text { for } \quad \theta \in\left[0, \theta_{i^{\prime} f}^{*}\right] \subset\left[0, \theta_{i f}^{*}\right],
$$

we then deduce that $\phi_{i}\left(\theta_{i^{\prime} f}\right) \leq 0$ (equality holds when $R_{i f}=1$ ). The unique critical point $\theta_{c}^{*}$ of $\phi_{i}(\theta)$ is found to be

$$
\theta_{c}^{*}=\left(\frac{b_{i}}{\beta a_{i}}\right)^{\frac{1}{\beta-1}} .
$$

It is quite straightforward to show that $0<\theta_{c}^{*}<\theta_{i^{\prime} f}^{*}$ and $\phi^{\prime \prime}\left(\theta_{c}^{*}\right)<0$ so that $\theta=\theta_{c}^{*}$ is a local maximum point (see the three lower curves in Figure 1). The function $\phi_{i}(\theta)$ has at least one root within $\left(0, \theta_{i^{\prime} f}^{*}\right)$ if and only if

$$
\phi\left(\theta_{c}^{*}\right)=\left(\frac{b_{i}}{\beta a_{i}}\right)^{\frac{1}{\beta-1}}\left(b_{i}-\frac{b_{i}}{\beta}\right)-1>0 .
$$

Rearranging terms in the above inequality, we obtain

$$
R_{i f}<q\left(\bar{R}_{i}\right)=\left[\frac{1}{\beta}\left(\frac{\bar{R}_{i}^{\beta}-1}{\bar{R}_{i}-1}\right)\right]^{\frac{1}{\beta-1}} .
$$

Next, it is necessary to show that $q\left(\bar{R}_{i}\right)>1$; otherwise, the condition $1 \leq R_{i f}<q\left(\bar{R}_{i}\right)$ does not hold. It suffices to show that $q(x)>1$ for $x>1$ and $\beta>1$. First, we observe that

$$
q(x)>1 \Leftrightarrow g(x)=x^{\beta}-1-\beta x+\beta>0 .
$$

Since $g(1)=0$ and $g^{\prime}(x)>0$ for $x>1$ and $\beta>1$, hence $g(x)>0$ and in turn $q(x)>1$. Now, under negative externalities, we have $\bar{R}_{i}>1$ and so $q\left(\bar{R}_{i}\right)>1$. 
Combining (i) and (ii), we deduce that $\theta_{i p}^{*}$ exists if and only if

$$
0<R_{i f}<q\left(\bar{R}_{i}\right)
$$

To prove Corollary 1 , we use symmetry to deduce that $\theta_{i^{\prime} p}^{*}$ exists if and only if $0<R_{i^{\prime} f}<q\left(\bar{R}_{i^{\prime}}\right)$, which is equivalent to $R_{i f}>\frac{1}{q\left(\bar{R}_{i^{\prime}}\right)}$ since $R_{i^{\prime} f}=$ $\frac{1}{R_{i f}}$. Combining this with the result from Proposition $1, \theta_{i p}^{*}$ and $\theta_{i^{\prime} p}^{*}$ both exist if and only if $\frac{1}{q\left(\bar{R}_{i^{\prime}}\right)}<R_{i f}<q\left(\bar{R}_{i}\right)$.

Suppose $R_{i f} \geq q\left(\bar{R}_{i}\right)$, then $\theta_{i p}^{*}$ does not exist. However, since

$$
R_{i^{\prime} f}=\frac{1}{R_{i f}} \leq \frac{1}{q\left(\bar{R}_{i}\right)}<1
$$

we deduce that $\theta_{i^{\prime} p}^{*}$ exists. Interchanging $i^{\prime}$ with $i$ in the above arguments, when $R_{i f} \leq \frac{1}{q\left(\bar{R}_{i^{\prime}}\right)}$, we deduce that $\theta_{i p}^{*}$ exists but not $\theta_{i^{\prime} p}^{*}$. Hence, the results in Corollary 2 are obtained.

The three inequalities in Corollary 3 are derived from negative externalities and existence conditions of both $\theta_{i p}^{*}$ and $\theta_{i^{\prime} p}^{*}$ :

$$
R_{i^{\prime} f}<q\left(\bar{R}_{i^{\prime}}\right), \quad R_{i f}<q\left(\bar{R}_{i}\right) \text { and } \quad \bar{R}_{i^{\prime}}>1
$$

The first two inequalities are obtained from the main result of Proposition 1. The last inequality arises as a derived condition of negative externalities for Firm $i^{\prime}$. This is seen as one of the necessary conditions for the existence of $\theta_{i^{\prime} p}^{*}$. It is straightforward to show that

$$
\begin{aligned}
R_{i^{\prime} f}<q\left(\bar{R}_{i^{\prime}}\right) & \Leftrightarrow R_{i \ell}>\frac{R_{i f}}{\bar{R}_{i}} q^{-1}\left(\frac{1}{R_{i f}}\right) \\
\bar{R}_{i^{\prime}}>1 & \Leftrightarrow R_{i \ell}>\frac{R_{i f}}{\bar{R}_{i}} .
\end{aligned}
$$




\section{Appendix C - Proof of Proposition 2}

Given $R_{i f}<1$, which is equivalent to $R_{i^{\prime} f}>1$, the two functions $\phi_{i}(\theta)$ and $\phi_{i^{\prime}}(\theta)$ are defined over $\left[0, \theta_{i f}^{*}\right]$. From Proposition 1, we obtain $\phi_{i}\left(\theta_{i f}^{*}\right)>0$ and $\phi_{i^{\prime}}\left(\theta_{i f}^{*}\right)<0$. Also, from $R_{i \ell}>1$, we obtain $\frac{d \phi_{i}}{d \theta}(0)<\frac{d \phi_{i^{\prime}}}{d \theta}(0)$. The five possibilities of how the curve of $\phi_{i}(\theta)$ intersects that of $\phi_{i^{\prime}}(\theta)$ are shown in Figures $3 \mathrm{a}-3 \mathrm{e}$. The relative magnitude of the roots of $\phi_{i}(\theta)$ and $\phi_{i^{\prime}}(\theta)$ can be deduced readily from Figures $3 \mathrm{a}-3 \mathrm{e}$.

\section{Appendix D - Proof of Proposition 3}

The condition $\theta_{i \ell}^{*} \leq \theta_{i^{\prime} p}^{*}$ is equivalent to $\left\{\phi_{i^{\prime}}\left(\theta_{i \ell}^{*}\right) \leq 0\right.$ and $\left.\frac{d \phi_{i^{\prime}}}{d \theta}\left(\theta_{i \ell}^{*}\right)>0\right\}$, that is, the point $\left(\theta_{i \ell}^{*}, \phi_{i^{\prime}}\left(\theta_{i \ell}^{*}\right)\right)$ does not go above the $\theta$-axis and it lies on the increasing part of $\phi_{i^{\prime}}(\theta)$. It can be shown that

$$
\begin{aligned}
& \phi_{i^{\prime}}\left(\theta_{i \ell}^{*}\right) \leq 0 \quad \Leftrightarrow \quad \beta\left(b_{k} \theta_{i \ell}^{*}-1\right) \leq \beta a_{k} \theta_{i \ell}^{* \beta} \\
& \frac{d \phi_{i \prime}}{d \theta}\left(\theta_{i \ell}^{*}\right)>0 \quad \Leftrightarrow \quad \beta a_{k} \theta_{i \ell}^{* \beta}<b_{k} \theta_{i \ell}^{*} .
\end{aligned}
$$

Combining the above two inequalities, we obtain

$$
\beta\left(\frac{\theta_{i \ell}^{*}}{\theta_{i^{\prime} \ell}^{*}}-1\right) \leq \beta\left(\frac{\theta_{i \ell}^{*}}{\theta_{i^{\prime} \ell}^{*}}-\frac{\theta_{i \ell}^{*}}{\theta_{i^{\prime} f}^{*}}\right)\left(\frac{\theta_{i \ell}^{*}}{\theta_{i f}^{*}}\right)^{\beta-1}+\left(\frac{\theta_{i \ell}^{*}}{\theta_{i^{\prime} f}^{*}}\right)^{\beta}<1 .
$$

Rearranging the terms, we obtain the result in Proposition 3. 


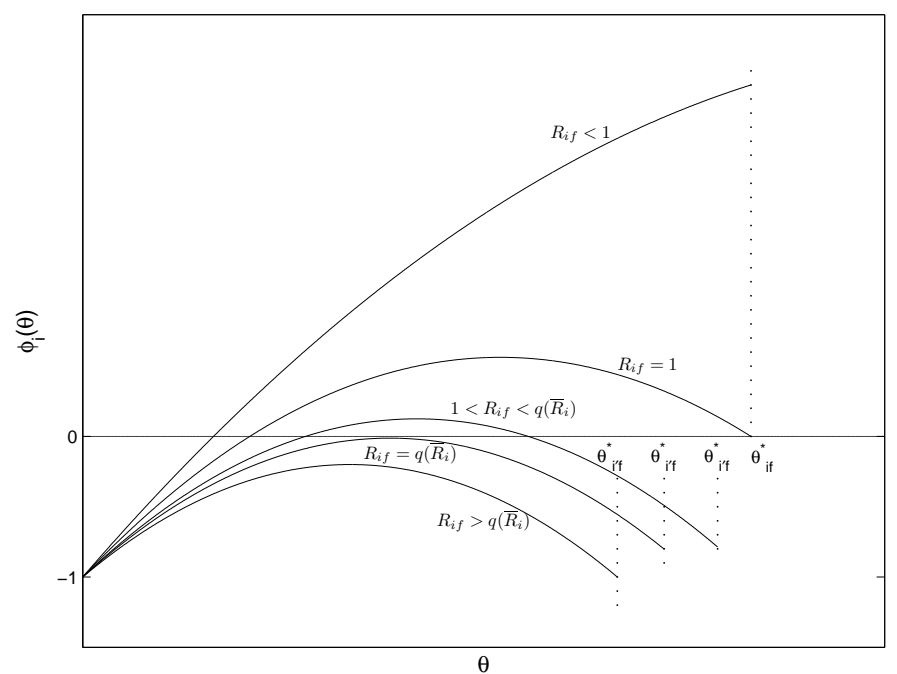

Fig. 1 Behaviors of $\phi_{i}(\theta)$ within the domain $\left[0, \min \left(\theta_{i f}^{*}, \theta_{i^{\prime} f}^{*}\right)\right]$ under varying values of $R_{i f}$. When $0<R_{i f}<q\left(\bar{R}_{i}\right), \phi_{i}(\theta)$ has at least one root so that the pre-emptive threshold $\theta_{i p}^{*}$ exists.

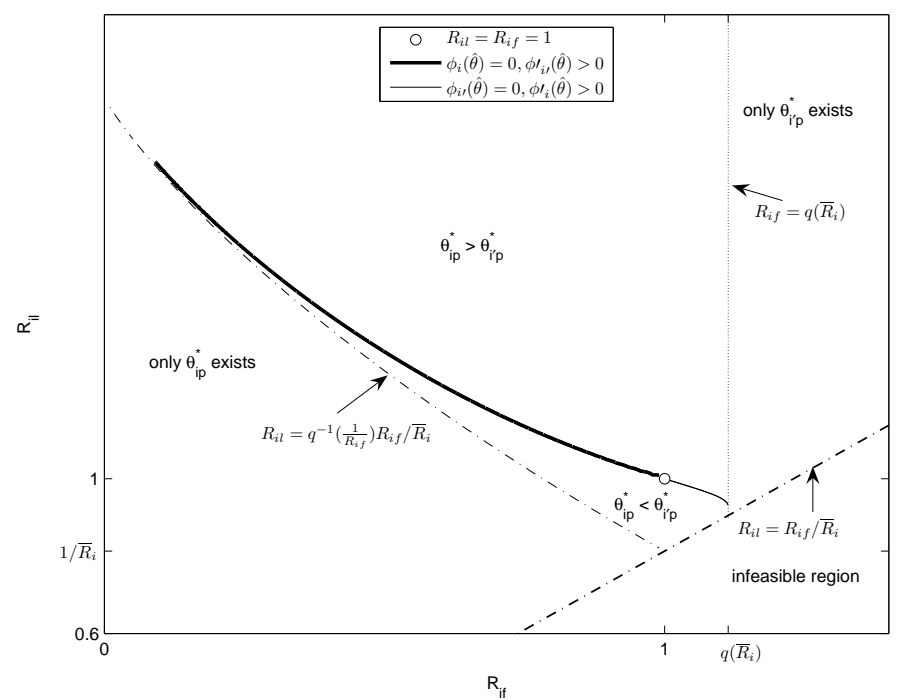

Fig. 2 Characterization of the relative magnitude of the pre-emptive thresholds, $\theta_{i p}^{*}$ and $\theta_{i^{\prime} p}^{*}$, in the $R_{i f}-R_{i \ell}$ plane. For a fixed value of the parameter $\bar{R}_{i}$, the region where both pre-emptive thresholds exist is bounded by $R_{i f}<q\left(\bar{R}_{i}\right), R_{i \ell}>q^{-1}\left(\frac{1}{R_{i f}}\right) R_{i f} / \bar{R}_{i}$ and $R_{i \ell}>R_{i f} / \bar{R}_{i}$. Under negative externalities, the feasible region is given by $R_{i \ell}>R_{i f} / \bar{R}_{i}$. 


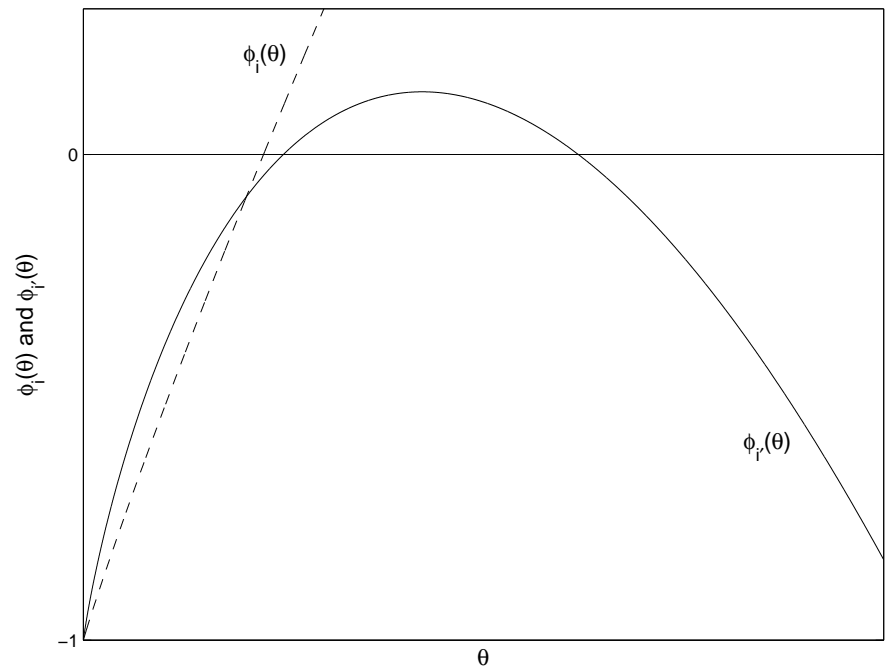

Fig. 3a $\phi_{i}(\widehat{\theta})<0$ and $\frac{d \phi_{i^{\prime}}}{d \theta}(\widehat{\theta})>0$ implying $\theta_{i p}^{*}<\theta_{i^{\prime} p}^{*}$.

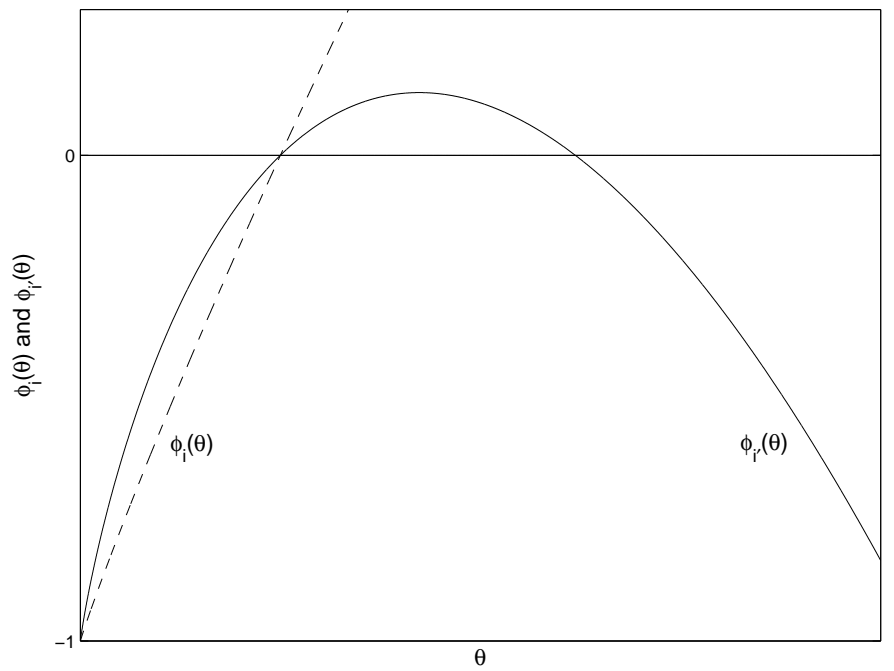

Fig. 3b $\phi_{i}(\widehat{\theta})=0$ and $\frac{d \phi_{i^{\prime}}}{d \theta}(\widehat{\theta})>0$ implying $\theta_{i p}^{*}=\theta_{i^{\prime} p}^{*}$. 


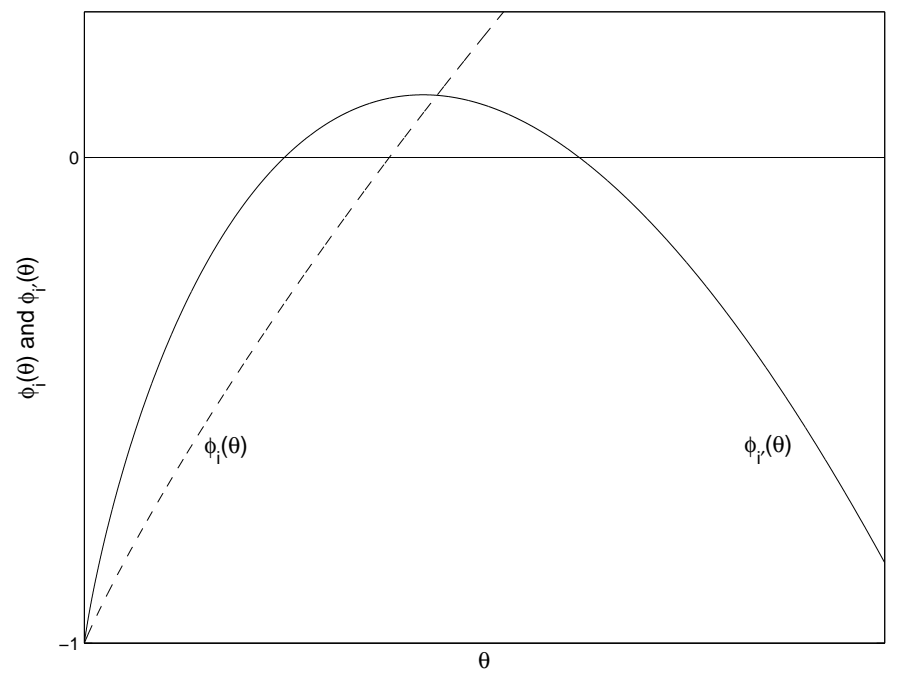

Fig. 3c $\phi_{i}(\widehat{\theta})>0$ and $\frac{d \phi_{i^{\prime}}}{d \theta}(\widehat{\theta})$ can be positive or negative, implying $\theta_{i p}^{*}>$ $\theta_{i^{\prime} p}^{*}$

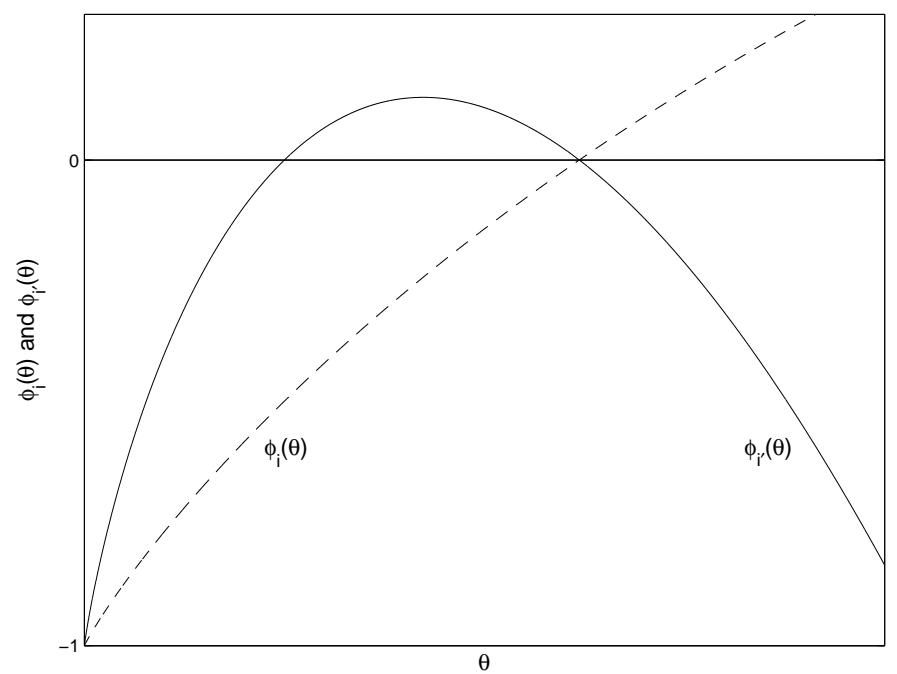

Fig. 3d $\quad \phi_{i}(\widehat{\theta})=0$ and $\frac{d \phi_{i^{\prime}}}{d \theta}(\widehat{\theta})<0$ implying $\theta_{i p}^{*}>\theta_{i^{\prime} p}^{*}$ 


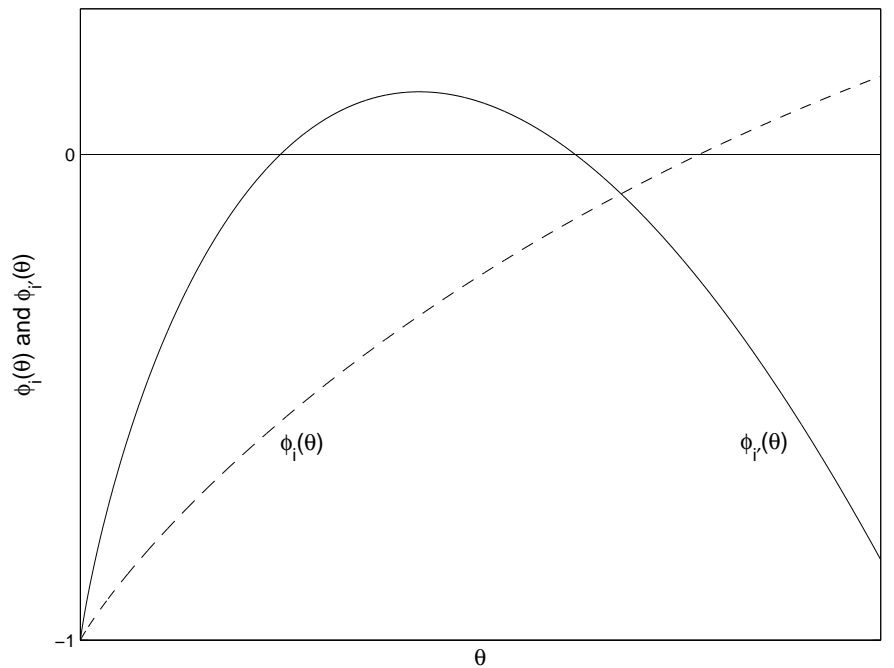

Fig. 3e $\quad \phi_{i}(\widehat{\theta})<0$ and $\frac{d \phi_{i^{\prime}}}{d \theta}(\widehat{\theta})<0$ implying $\theta_{i p}^{*}>\theta_{i^{\prime} p}^{*}$.

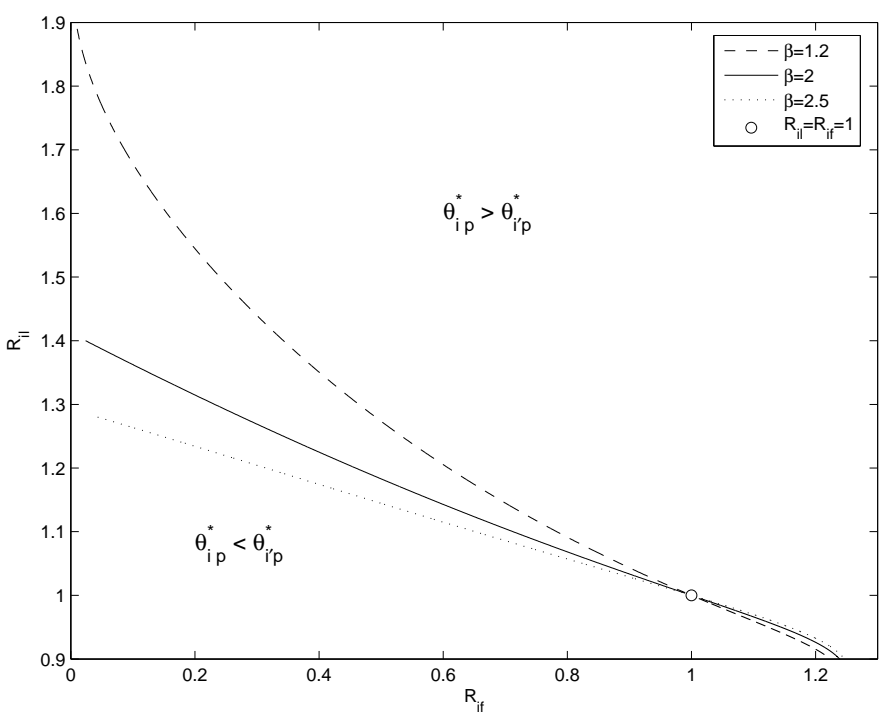

Fig. 4 The dependence of the separating curve: $\left\{\left(R_{i f}, R_{i \ell}\right): \theta_{i p}^{*}=\theta_{i^{\prime} p}^{*}\right\}$ on the parameter $\beta$. 


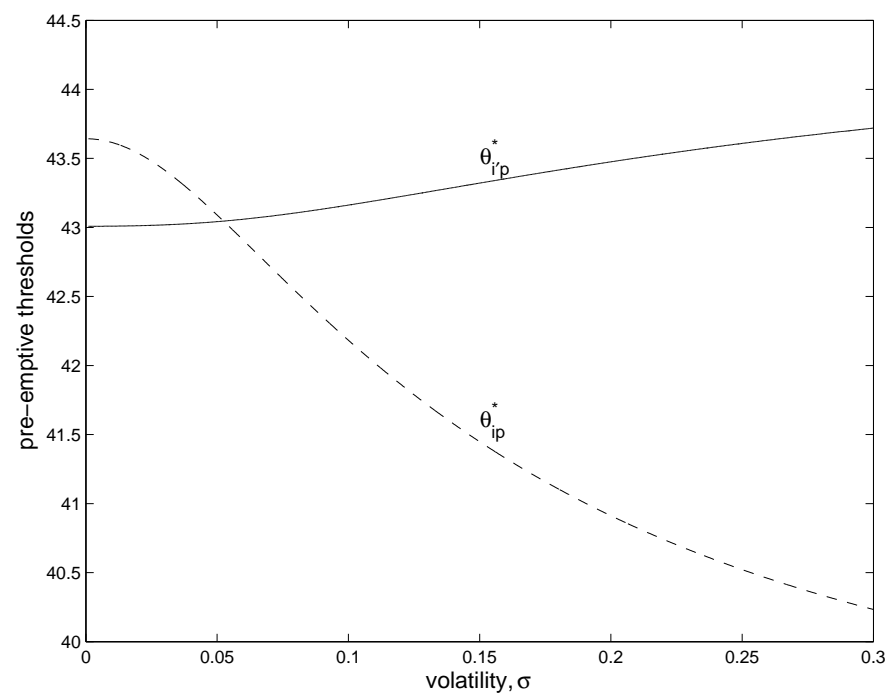

Fig. 5 With increasing volatility $\sigma, \theta_{i^{\prime} p}^{*}$ increases while $\theta_{i p}^{*}$ decreases. The parameter values used in the calculations: $R_{i f}=\frac{69}{100}, R_{i \ell}=\frac{57}{50}, \bar{R}_{i}=\frac{69}{57}$ (corresponding to $\left.\theta_{i^{\prime} \ell}^{*}=50, \theta_{i \ell}^{*}=57, \theta_{i f}^{*}=69, \theta_{i^{\prime} f}^{*}=100\right), r=4 \%$ and $\mu=1.6 \%$. 